\title{
Research of Improved Ant Colony Hybrid Algorithm
}

\author{
Li Shijun ${ }^{1, \mathrm{a}}$, Han $\mathrm{Yu}^{1, \mathrm{~b}}$, Gu Hongjun ${ }^{1, \mathrm{c}}$, Gong He $\mathrm{He}^{1, \mathrm{~d}}$, Li Jian ${ }^{1, \mathrm{el}}$ \\ ${ }^{1}$ College of Information \&Technology, Jilin Agricultural University, Changchun 130118, China. \\ a452835889@qq.com, b372600730@qq.com, '330837495@qq.com \\ d29878671@qq.com, ${ }^{\mathrm{e}} 2312852319 @ q q . c o m$
}

\begin{abstract}
Keywords: ant colony algorithm, immune algorithm, artificial fish swarm algorithm, hybrid algorithm.
\end{abstract}

\begin{abstract}
In order to extend the application of ant colony algorithm (ACA), many scholars combined the ant colony algorithm with immune algorithm (IA) or other algorithms to solve the problem of slow convergence. To fully solve the too long search time, easily falling into local optimization, slow convergence and some other defects, the immune algorithm and artificial fish swarm algorithm (AFSA) combine with the ant colony algorithm, and the ant colony hybrid algorithm is proposed. Then by solving the traveling salesman problem (TSP), the new algorithm is simulated, and the results show that improving algorithm is effective and feasible.
\end{abstract}

\section{Introduction}

The ant colony algorithm (ACA) was first proposed by Italy scholar Dorigo M. and others in 1991, a novel simulated evolutionary algorithm, ants search for a path through the secretion of pheromone scattered on its path. Then the ants randomly choose a road that didn't pass, release pheromone about the length of this path. But the amount of information released is inversely proportional to the length of the path, which likely to choose the path of a larger amount of information, it's a positive feedback mechanism. The best path is the amount of information that is getting bigger and bigger, the amount of information on the other path is gradually reduced, ants eventually find optimal path. By simulating ants' behavior such as foraging, assignment and building the graveyard, we put forward ACA. It's easy to combine with other algorithms, and it has a strong robustness and excellent distributed computer system, and it's easy to combine with other algorithms. This algorithm achieved good effect in the academic field, the problems such as function optimization, combination optimization, data mining, network routing, etc. ACA became a hot spot for many scholars to analyze the optimization algorithm, it has unique and widely ability to solve problems. Improved the algorithm itself, and combined with other algorithms, applied to many of the actual field of which.

\section{Basic principles of ant colony algorithm}

$\tau_{i j}(t)$ First, solving the TSP problem as an example, the specific implementation steps of the basic ACA are as follows. Giving $n$ cities and two of the distance between the two cities, the requirements to determine a passed through each city only once in the shortest path. In order to simulate the behavior of real ants, we introduced the following notation; $m$ is the number of ants. $d_{i j}(i, j=1,2, \ldots, n)$ represents the distance between $i$ and $j$ in the city, represents the amount of information remaining on the path between $i$ and $j$ in the $t$ city, it's used to simulate the pheromone concentration allowed $_{k} \operatorname{tabu}_{k} p_{i j}^{k}(t)$ of the actual ants.. When initialized, $m$ ants were placed randomly on different cities, giving the $\tau_{i j}(0)$ amount of information was on each side. Each ant $k$ of that the first element was assigned to the city where it was located. indicate that the ant $k$ was transferred from city $i$ to city $j$ probability time, using formula (1). indicates that ant $k$ allows to chose the city in the next step; $\alpha$ as information heuristic factor, indicates relative locus importance. It reflects that ants accumulated information in the movement to play the role for the ant movement; 
$\beta$ as expectation heuristic factor, indicates relative visibility importance, it reflects the importance degree of heuristic information when ants chose the path in the movement.

$$
p_{i j}^{k}(t)=\left\{\begin{array}{l}
\frac{\tau_{i j}^{\partial}(t) \eta_{i j}^{\beta}(t)}{\sum_{\substack{s \in \text { allowed }_{k} \\
0,}} \tau_{i s}^{\partial}(t) \eta_{i s}^{\beta}(t)}, \text { if } \mathrm{j} \in \text { allowed }_{k} \\
\text { else }
\end{array}\right.
$$

After $n$ moments, ant $k$ walked through all the cities, completed a cycle. Then updated the amount of information on each path by the formula (2).

$$
\begin{gathered}
\Delta \tau_{i j} \\
\tau_{i j}(t+n)=(1-\rho) \tau_{i j}(t)+\Delta \tau_{i j}(t)
\end{gathered}
$$

Among, calculated by the formula (3), it represents the amount of information on the $k$ ant in the path $(i, j)$ in the loop. The calculation method is based on the calculation model, in the most commonly used Ant-Cycle model, using formula (4), $Q$ represents the pheromone intensity, it affects the convergence rate of the algorithm to some extent. $L_{k}$ represents the total length of the path of the $k$ ant in this cycle.

$$
\begin{aligned}
\Delta \tau_{i j}(t) & =\sum_{k=1}^{m} \Delta \tau_{i j}^{k}(t) \\
\Delta \tau_{i j}^{k}(t) & =\left\{\begin{array}{l}
\frac{Q}{L_{k}}, \text { If the K only ants in this cycle through }(\mathrm{i}, j) \\
0, \text { otherwise }
\end{array}\right.
\end{aligned}
$$

\section{Combination of ant colony algorithm and immune algorithm.}

The basic idea of the combination of ant colony algorithm and immune algorithm. Combing IA with ACA, used ACA to solve the problem as antigen, and the extraction of the vaccine to pheromone initialization, ACA produced antibodies to assign a value to a parameter, applied to the solution of specific problems, the obtained results as the current anti body fitness value by inoculation of vaccine IA, crossover, mutation, affinity selection, retained to adapt good antibody, eliminated adaptation of antibody, the iterative, got the antibody in finally, the parameter ACA combination was obtained for the specific problem. Algorithm by updating based on affinity, thus effectively prevents the 'premature' problem, led the search process to the global optimum. The initial value of pheromone was extracted by the extraction mechanism of vaccine, avoided the randomness of the initial solution. By using the vaccination mechanism, crossover and mutation to accelerate the convergence speed. ACA and IA is called immune ant colony algorithm (IAACA).

The basic steps of the immune ant colony algorithm. Immune ant colony algorithm flow chart is shown in Fig.1. 


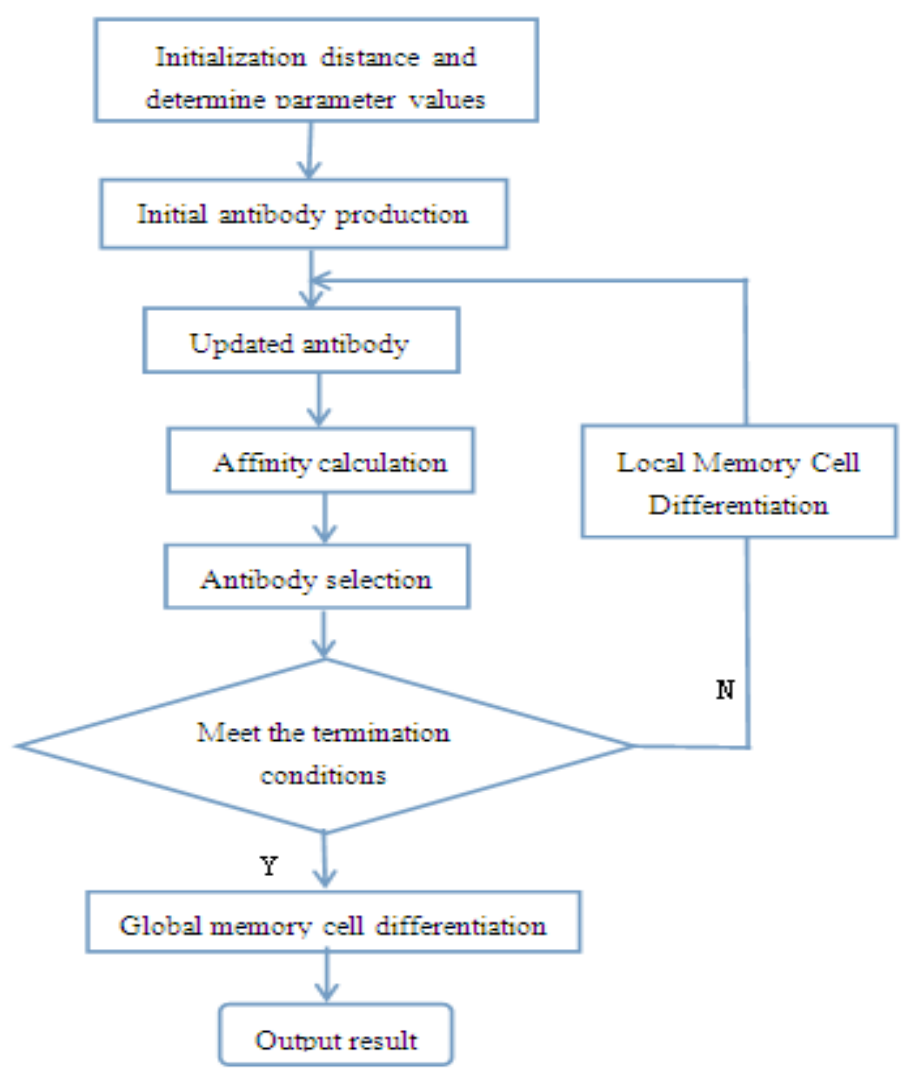

Fig. 1 Immune algorithm flow chart

\section{Combination of artificial fish swarm algorithm and immune ant colony algorithm}

The basic idea of the hybrid algorithm (AFSA-IAACA) based on artificial fish swarm algorithm and ant colony immune algorithm. AFSA has the advantages of fast convergence speed, we will added to every iterative process of immune ant colony algorithm to accelerate the convergence speed of ant colony algorithm, and depending on the foraging behavior of AFSA to help improve IAACA to jump out of local optimum.

AFSA-IAACA for the TSP problem of a detailed improvement strategy and algorithm detailed steps: Step1 At the initial $\mathrm{t}=0, \mathrm{~m}$ ants were randomly placed in the $\mathrm{n}$ cities, each path initial pheromone concentration is $\tau_{i j}(0)=$ const.

Step2 Ants calculated transfer probability by the formula (1), selected to project transition path. Then calculated the path congestion at that time $q_{i j}$ by the formula (5). If $q_{i j}\langle\delta(t)$ indicated that path is not too crowded, ants chose the path to transfer from position $i$ to position $j$. Otherwise, the path was too crowded, the ant selected a path of transfer in the feasible neighborhood to randomly. Among them, $\quad \delta(t)$ is congestion threshold in $t$ time, updated type by the formula (6). Among them, $c$ is the threshold coefficient of variation.

$$
\begin{aligned}
& q_{i j}=\frac{2 \tau_{i j}(t)}{\sum_{i \neq j} \tau_{i j}(t)} \\
& \delta(t)=1-e^{-c t}
\end{aligned}
$$

Step3 After $n$ moment, the $k$ ant went all Cities to complete a cycle. Then updated the information on each path by the formula (2).

Step4 Repeated formula (1) and (2), until the m ants chose the same path or reach the specified maximum. 


\section{Simulation Results and Analysis}

Table1 Comparison of experimental results

\begin{tabular}{lllllll}
\hline & \multicolumn{2}{c}{ (ACA) } & \multicolumn{2}{c}{ (IAACA) } & \multicolumn{2}{l}{ (AFSA-IAACA) } \\
\cline { 2 - 7 } $\begin{array}{l}\text { number } \\
\text { of cities }\end{array}$ & $\begin{array}{l}\text { average } \\
\text { iteration } \\
\text { number }\end{array}$ & Optimum & $\begin{array}{l}\text { average } \\
\text { iteration } \\
\text { number }\end{array}$ & $\begin{array}{l}\text { Optimum } \\
\text { solution }\end{array}$ & $\begin{array}{l}\text { average } \\
\text { iteration } \\
\text { number }\end{array}$ & $\begin{array}{l}\text { Optimum } \\
\text { solution }\end{array}$ \\
10 & 103 & 2.708617 & 98 & 2.708617 & 91 & 2.708617 \\
30 & 728 & 423.631000 & 596 & 423.631000 & 547 & 423.631000 \\
50 & 1465 & 429.543000 & 962 & 427.865000 & 715 & 427.653 .000 \\
75 & 2103 & 569.783000 & 1421 & 551.649000 & 892 & 541.443000 \\
\hline
\end{tabular}

The results show that the AFSA-IAACA algorithm proved its feasibility, effectiveness and convergence by application and simulation experiment in the TSP problem. The algorithm will AFSA added to each iterative process of IAACA, taking advantage of AFSA with fast convergence which accelerate the convergence speed of ACA and foraging behavior of AFSA could help improved the ability of ACA to jump out of local optimum. By doing this, we can reduce not only the number of invalid search, but also the algorithm into the local optimal solution, improve the ability and convergence speed of the algorithm.

\section{Conclusions}

ACA has some problems, such as premature convergence, slow convergence, the combination of ACA and IA is an effective method to solve these defects. Then combine the IAACA and AFSA to solve these defects that long search time and easily fall into local optimization, and to improve the ability to jump extreme, and significantly improve the accuracy of the algorithm. The simulation experiment was carried out by solving the TSP problem, and results show that the improved algorithm is effective and feasible.

\section{Acknowledgments}

The authors wish to express their gratitude to the projects: Jilin Province Economic Structural Adjustment Leading Fund Special Project (No. 2014Y108) and Changchun City Science and Technology Plan Project (No. 14nk029), Key Tackling Item of Jilin Province Science \& Technology Department (No. 20140204045NY), Design of Standardized Breeding System for Rabbits Based on Internet of Things from Education Department of Jilin Province, Changchun City Science and Technology Plan Project (No. 13KG71), for their generous support of this work.

\section{References}

[1] Gu Mingjia, Xuan Shi bin, Lian Kanchao, et al. QoS routing algorithm based on combination of modified ant colony algorithm and artificial fish swarm algorithm, Computer technology and development, 2009, pp.145-148.

[2] He Yijun, Chen Dezhao. The construction and application of ant colony algorithm for multi-objective optimization, High technology Communication, Beijing, 2006, pp.1241-1245.

[3] Cai Lijun, Jiang Linbo, Yi Ye Qing. Gene selection based on ant colony optimization algorithm, Calculation and Application Research. Beijing, 2008, pp.2754-2756.

[4] Duan Haibin. Ant colony algorithm and its application, Science Press, Beijing, 2005.

[5] Dasgupta D. Advances in artificial immune systems, IEEE Computational Intelligence Magazine, Beijing, 2006, pp.40-49. 
[6] Jiang Xinzi, Tong Kezong, Gao Shang. Hybrid algorithm of ant colony algorithm with immune algorithm, Science Technology and Engineering, Beijing, 2008, pp.1328-1333. 